\title{
Incidence and outcomes of intracerebral haemorrhage with mechanical compression hydrocephalus
}

Bryce Owen (D) , ${ }^{1}$ Omar Akbik, ${ }^{2}$ Michel Torbey, ${ }^{3}$ Herbert Davis, ${ }^{4}$ Andrew P Carlson ${ }^{2}$

To cite: Owen B, Akbik 0 , Torbey M, et al. Incidence and outcomes of intracerebral haemorrhage with mechanical compression hydrocephalus. Stroke \& Vascular Neurology 2021;6: e000401. doi:10.1136/ svn-2020-000401

Received 16 April 2020 Revised 10 July 2020 Accepted 14 August 2020

Published Online First

8 January 2021

\section{Check for updates}

(C) Author(s) (or their employer(s)) 2021. Re-use permitted under CC BY-NC. No commercial re-use. See rights and permissions. Published by BMJ.

${ }^{1}$ School of Medicine, University of New Mexico School of Medicine, Albuquerque, New Mexico, USA

${ }^{2}$ Neurosurgery, University of New Mexico, Albuquerque, New Mexico, USA

${ }^{3}$ Neurology, University of New Mexico, Albuquerque, New Mexico, USA

${ }^{4}$ Division of Epidemiology, Biostatistics, and Preventive Medicine, University of New Mexico, Albuquerque, New Mexico, USA

Correspondence to Dr Andrew P Carlson; AndrewCarlson@salud.unm.edu

\section{ABSTRACT}

Introduction Intracerebral haemorrhage (ICH) within deep structures adjacent to the third ventricle is associated with worse outcomes when compared with lobar ICH due to the critical role of deep nuclei in normal neurological functioning. New evidence suggests another contributing factor to poor outcome is obstruction of cerebrospinal fluid outflow by clot burden causing mechanical compression of the third ventricle. The authors reviewed the incidence and outcomes of mechanical compression ICH in order to identify this high-risk group which may potentially benefit from minimally invasive evacuation.

Methods Patients with spontaneous, non-traumatic, supratentorial ICH were identified retrospectively over a 30-month period. CT imaging was reviewed to assess location of the $\mathrm{ICH}$, volume of the $\mathrm{ICH}$, presence of hydrocephalus requiring external ventricular drain (EVD) placement, and time to clearing of the third ventricle. Hydrocephalus was then categorised as due to 'primarily intraventricular haemorrhage (IVH)', 'primarily mechanical compression' or 'mixed'. Functional outcomes at discharge were assessed using the modified Rankin Score (mRS).

Results 287 patients met inclusion criteria, of which 39 (13.5\%) patients developed hydrocephalus that required EVD. EVD patients had significantly higher $\mathrm{mRS}$ at discharge $(p \leq 0.001)$ when compared with the non-EVD group. Lobar location was associated with lower odds of poor outcome compared with thalamic location (OR $0.107-0.560)$. Mechanical compression hydrocephalus was associated with poor outcome when compared with the primary IVH hydrocephalus subgroup $(p=0.037)$ as well as longer time to clearing of the third ventricle $(p=0.006)$. Conclusions Mechanical obstruction requiring EVD occurs in approximately $(21 / 287) 7.3 \%$ of all patients with spontaneous supratentorial ICH. It is unknown if the worse morbidity in these subjects is purely related to damage to deep structures surrounding the third ventricle or if secondary damage from hydrocephalus could be mitigated with targeted minimally invasive clot evacuation.

\section{INTRODUCTION/BACKGROUND}

Nearly 800000 people have a stroke annually in the USA, of which $87 \%$ are ischaemic and $13 \%$ are haemorrhagic. ${ }^{1}$ Even though intracerebral haemorrhage (ICH) is less common, it associated with higher rates of mortality and morbidity compared with ischaemic stroke. ${ }^{2}$ More than one-third of individuals die in the first month after ICH, and patients who survive have a continuing elevated risk of death compared with matched individuals from the general population. ${ }^{3}$ Despite advances in the treatment of cerebral ischaemic infarction and subarachnoid haemorrhage in recent years, there remain few therapies of proven benefit in improving outcome after ICH. The current American Heart Association guidelines endorsed by the American Association of Neurological Surgeons and the Congress of Neurological Surgeons state that for most patients with supratentorial ICH, the usefulness of surgery is not well established. ${ }^{4}$

Surgical evacuation of ICH has been a widely debated and studied topic for over half a century. ${ }^{56}$ The Surgical Treatment for IntraCerebral Hemorrhage (STICH) trial $^{7}$ evaluated the efficacy of early surgery versus initial conservative treatment in patients with spontaneous intracerebral haematoma. The authors demonstrated no difference in outcome between early surgery versus initial conservative treatment. STICH $\mathrm{II}^{8}$ targeted patients most likely to benefit based on STICH subgroup analysis and demonstrated lower mortality in the early surgery group, but no functional benefit. It is important to note that surgery was still used commonly as a life saving measure in both of these studies; however, an initial strategy of surgery did not seem to be effective to improve outcomes. In more stable patients who are less likely to deteriorate, minimally invasive approaches as an initial strategy have been increasingly studied. The Minimally Invasive Surgery plus Rt-PA for ICH Evacuation (MISTIE) studies are the largest such studies comparing medical management to stereotactic ICH drainage with alteplase dosing over 72 hours. ${ }^{69}$ Ultimately, MISTIE III demonstrated improvement in mortality without functional improvement in the surgical arm. Other minimally invasive techniques have shown early promise and pivotal trials are ongoing. ${ }^{10} 11$

Primarily intraventricular haemorrhage (IVH) is a related condition but has historically been studied separately. The Clot Lysis: 


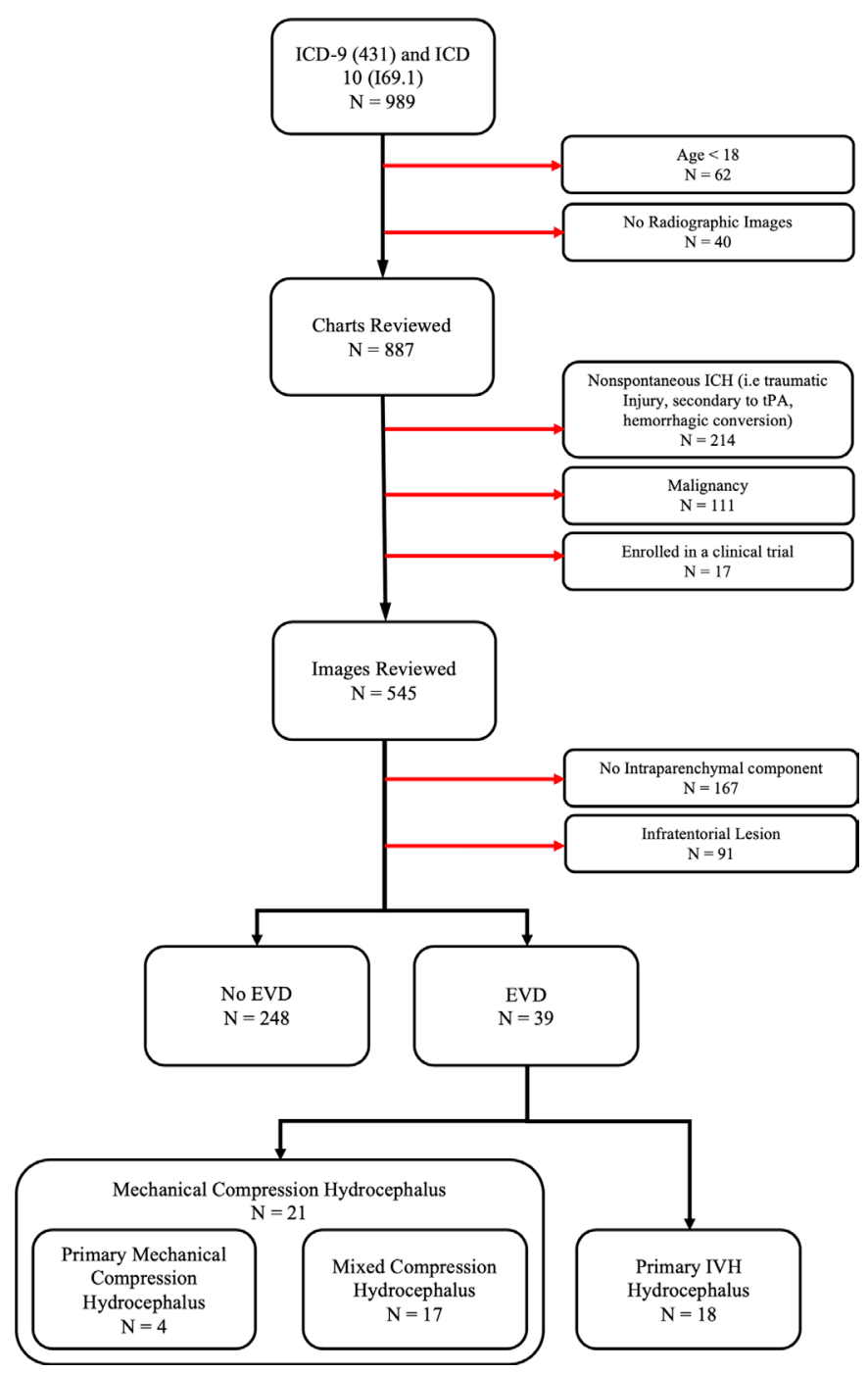

Figure 1 Exclusion criteria. EVD, external ventricular drain; ICD, International Classification of Diseases; ICH, intracerebral haemorrhage; IVH, intraventricular haemorrhage; tPA, tissue Plasmonogen Avtivator.

Evaluating Accelerated Resolution of Intraventricular hemorrhage (CLEAR) trials $^{12}$ studied the effect of intraventricular alteplase versus saline in patients requiring external ventricular drain (EVD), however, failed to demonstrate a significant improvement in functional outcome or other measures with alteplase. A posthoc analysis of CLEAR patients demonstrated that there were significantly worse outcomes in patients with IVH treated with alteplase if the hydrocephalus was primarily from mechanical compression due to thalamic haemorrhage, whereas if the hydrocephalus was primarily from IVH, alteplase was associated with better outcomes. ${ }^{13}$

Though these studies have significantly expanded our knowledge of ICH/IVH management, this important group of patients with hydrocephalus from mechanical compression identified by Ullman et al may have been largely missed in both studies. MISTIE excluded patients with significant hydrocephalus from IVH and CLEAR excluded ICH larger than $30 \mathrm{~cm}^{3}$. The goal of this study was to characterise this population and determine the overall incidence and outcomes compared with other patients with ICH and IVH, as well as the ICH population as a whole. As rising evidence suggests, this study seeks to investigate if patients with mechanical compression have worse outcomes than other patients with ICH without such mechanical compression. Presented here is a 'proof of concept' technical feasibility report regarding targeted minimally invasive ICH evacuation for a patient with mechanical compression.

\section{METHODS}

All patients admitted to the (INSTITUTION BLINDED) Hospital from May 2015 to December 2017 with ICD-10 code I61.9 (non-traumatic ICH) or ICD-9 code 431 (ICH) were retrospectively identified. Patient identifiers were not used and a waiver for consent was granted. Exclusion criteria are detailed in figure 1. Initial CT imaging was reviewed and ICH location was assigned as either thalamic, basal ganglia or lobar. ICH volumes were measured using the ABC/2 method. Presence of IVH was recorded as either present or not present. An ICH Score was calculated for each patient using the standard ICH scoring system. ${ }^{14}$ Infratentorial haemorrhage was an exclusion criteria for this study; thus, the maximum possible ICH Score was 5.

Provider discretion was used in the decision to place an EVD, factors that were considered include radiographic evidence of hydrocephalus, decreased level of consciousness or significant IVH. The placement of a ventriculoperitoneal shunt for persistent hydrocephalus was done after multiple attempts are made to wean the EVD with failure determined by following intracranial pressure, clinical examination and radiographic ventricular size. Patients requiring an EVD were further categorised into subgroups based on characteristics of ICH at the point when hydrocephalus was first radiographically identified. No patients switched hydrocephalus category after initial assignment was made. Two authors reviewed all EVD patient CT scans and independently assigned each to a hydrocephalus category. In cases where there was a discrepancy, the scans were reviewed jointly, and a consensus was made. The primary IVH subgroup consisted of patients with hydrocephalus caused primarily by IVH volume resulting in occlusion of the third and fourth ventricles (figure 2A). The primary mechanical compression subgroup demonstrated ventricular occlusion via ICH mass effect alone with little intraventricular blood (figure 2B). The mixed subgroup consisted of patients with hydrocephalus caused by a combination of intraventricular blood as well as mass effect from ICH (figure 2C). Patients with mixed and primary mechanical compression hydrocephalus were combined for subgroup analysis into the mechanical compression group.

All patients in the EVD group were followed with serial CT studies. Opening pressure was noted at time of EVD placement. Glasgow Coma Scale was assessed at time of 


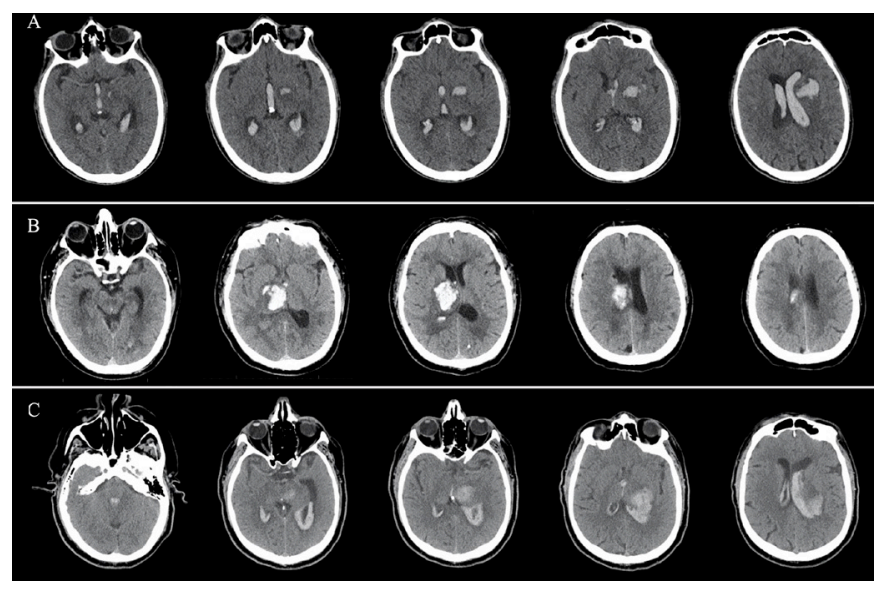

Figure 2 External ventricular drain subgroups. (A) 'Primary IVH subgroup' - primarily intraventricular haemorrhage (IVH) causing ventricular obstruction with distant intracerebral haemorrhage $(\mathrm{ICH})$ not compressing the ventricular system. (B) 'Primary mechanical compression subgroup' - primarily mechanical compression ICH showing minimal IVH present with direct clot burden obstructing ventricular outflow.

(C) 'Mixed subgroup'-mixed components of mechanical compression and IVH causing hydrocephalus.

presentation. Rates of meningitis, defined as one cerebrospinal fluid (CSF) culture with positive bacterial infection, were tracked in the EVD cohort. In addition to tracking presence of IVH, patients in EVD cohort were analysed for IVH volume using the modified Graeb Score. ${ }^{15}$ All patients who were given alteplase directly into the ICH were excluded from the review; however, alteplase was routinely given intraventricularly with the goal of clearing the third and fourth ventricles. The number of days with EVD were calculated by noting the first day of placement subtracted by the day the EVD was confirmed removed. The number of days to the radiographic opening of the third ventricle was determined by following serial CT examinations until patency of the third ventricle was evident. Patients who died, or were shunted before clearing of the third ventricle occurred, were excluded from analysis of this variable. Surgical evacuation of the ICH was performed in cases of life-threatening mass effect. Patient outcomes were measured using the modified Rankin Score (mRS) at discharge and scored on a scale from 0 to 6 . Due to the retrospective nature of this study, mRS was abstracted from multidisciplinary and clinical notes. Outcomes were dichotomised in the analysis to either 'good outcome' referring to a $\mathrm{mRS}$ of $0-3$, or 'poor outcome' indicating an mRS of 4-6. Withdrawal of care was noted as occurring or not occurring in all patients with in-hospital mortality. In the EVD cohort, the timing of the withdrawal of care was calculated as time from admission to time when family discussion occurred to withdraw care.

For univariate analysis, categorical variables were described as frequency rates and percentages. Continuous data were presented as means and ranges. Proportions for categorical variables were compared using the $\chi 2$ test, although the Fisher exact test was used where appropriate. Means for continuous variables were compared using independent group t tests when the data were normally distributed; otherwise, the Mann-Whitney test was used. A two-sided $\alpha$ of less than 0.05 was considered statistically significant. Multivariable logistic regression analysis was performed on statistically significant and clinically relevant univariate variables. Statistical analysis for these data was generated using SAS software, V.9.4 of the SAS System for Microsoft Windows (SAS Institute).

\section{RESULTS}

Nine hundred eighty-nine charts were found initially. After exclusions (figure 1), 287 patients were identified admitted between May 2015 and January 2018. Comparisons between the non-EVD group and the EVD group were analysed and are demonstrated in table 1.

There were $39(13.5 \%)$ patients who underwent placement of an EVD for treatment of hydrocephalus, with the remainder $(n=248860.4 \%)$ in the non-EVD group. Within the EVD subgroups (table 2), 18 (46.1\%) developed hydrocephalus from IVH, 4 (10.2\%) from primary mechanical compression and $17(43.6 \%)$ from mixed components.

The non-EVD group had a significantly higher average age compared with the EVD group (64.50 vs 57.69 $\mathrm{p}=0.007)$, although the portion of patients over 65 was non-significantly different between groups $(49.6 \%$ vs $35.9 \% \mathrm{p}=0.122)$. The EVD subgroups did not differ significantly in age $(p=0.267)$. The EVD group had significantly longer length of stay compared with the non-EVD group (18.92 vs $8.85 \mathrm{p} \leq 0.001$ ), with the portion of stays $\geq 7$ days also significantly larger in the EVD group $(47.9 \%$ vs $69.2 \% \mathrm{p}=0.014$ ). The EVD subgroup analysis did not show a significant difference between EVD groups and length of stay $(p=0.358)$. The EVD group had a significantly larger proportion of thalamic versus lobar haemorrhages compared with the non-EVD group $(p=0.001)$. Comparisons of the EVD subgroups demonstrated a significantly higher portion of thalamic versus lobar haemorrhages in the mechanical compression group compared with the IVH group $(\mathrm{p} \leq 0.001)$. The non-EVD group and EVD group had similarly sized ICH volumes $(40.3 \mathrm{~mL}$ vs 36.7 $\mathrm{mL} \mathrm{p}=0.521$ ), as well as the portion of patients with ICH volume $\geq 30 \mathrm{~mm}^{3}$ (39.5\% vs $\left.41.0 \% \mathrm{p}=0.862\right)$. The EVD subgroups did not differ significantly in ICH volume (42.1 $\mathrm{mL}^{3}$ vs $\left.30.6 \mathrm{~mL}^{3} \mathrm{p}=0.384\right)$. The modified Graeb Score was lower in the mechanical compression subgroup, although this did not reach significance $(p=0.058)$. The modified Graeb was lower in the EVD subgroup analysis of poor outcome $(p=0.01)$. The number of days to the radiographic opening of the third ventricle was significantly longer in the mechanical compression subgroup compared with the IVH subgroup (13.2 days vs 7.3 days $\mathrm{p}=0.006)$. The authors reviewed the rate of withdrawal of care between the non-EVD and EVD cohorts and found them to be non-significantly different $(16.5 \%$ vs $28.2 \%$ 
Table 1 Comparison of non-external ventricular drain (EVD) and EVD groups

\begin{tabular}{|c|c|c|c|}
\hline Variable* & $\begin{array}{l}\text { Non-EVD group } \\
(\mathrm{n}=248)\end{array}$ & $\begin{array}{l}\text { EVD group } \\
(n=39)\end{array}$ & $P$ value \\
\hline \multicolumn{4}{|l|}{ Demographics } \\
\hline Total patients & 248 & 39 & $\ldots$ \\
\hline Male sex & $122(49.2 \%)$ & $27(69.2 \%)$ & 0.020 \\
\hline \multicolumn{4}{|l|}{ Age, in years } \\
\hline Average age (years) & $64.50 \pm 14.82$ & $57.69 \pm 13.88$ & 0.007 \\
\hline Age $\geq 65$ years & $113(49.6 \%)$ & $14(35.90 \%)$ & 0.122 \\
\hline \multicolumn{4}{|l|}{ Length of hospital stay, in days } \\
\hline Average (range) & $8.85 \pm 9.91(0-81)$ & $18.92 \pm 15.09(0-57)$ & $<0.001$ \\
\hline$\geq 7$ days & $119(47.9 \%)$ & $27(69.2 \%)$ & 0.014 \\
\hline \multicolumn{4}{|l|}{$\mathrm{ICH}$ location } \\
\hline Basal ganglia versus thalamic & $50(20.2 \%)$ versus $40(16.1 \%)$ & $8(20.5 \%)$ versus $19(48.7 \%)$ & 0.018 \\
\hline Lobar versus thalamic & $158(63.7 \%)$ versus 40 (16.1\%) & $12(30.8 \%)$ versus $19(48.7 \%)$ & $<0.001$ \\
\hline Basal ganglia versus lobar & $50(20.2 \%)$ versus $158(63.7 \%)$ & $8(20.5 \%)$ versus $12(30.8 \%)$ & 0.117 \\
\hline \multicolumn{4}{|l|}{$\mathrm{ICH}$ Score } \\
\hline Score 2 versus $0-1$ & $44(17.7 \%)$ versus $153(61.7 \%)$ & $15(38.5 \%)$ versus 5 (12.8\%) & $<0.001$ \\
\hline Score 3 versus $0-1$ & $29(11.7 \%)$ versus $153(61.7 \%)$ & 16 (41.0\%) versus 5 (12.8\%) & $<0.001$ \\
\hline Score 4 versus $0-1$ & 22 (8.9\%) versus $153(61.7 \%)$ & $3(7.7 \%)$ versus $5(12.8 \%)$ & 0.080 \\
\hline \multicolumn{4}{|l|}{$\mathrm{ICH}$ volume, in $\mathrm{mm}^{3}$} \\
\hline Average (range) & $40.29 \pm 50.72(0.5-248)$ & $36.74 \pm 42.33(3.9-189)$ & 0.520 \\
\hline $\mathrm{ICH}$ volume greater than $30 \mathrm{~mm}^{3}$ & $98(39.5 \%)$ & $16(41.0 \%)$ & 0.862 \\
\hline Presence of IVH & $84(29.2 \%)$ & $39(100 \%)$ & $<0.001$ \\
\hline Modified Rankin Score at Discharge Score 4-6 & $129(52.0 \%)$ & $35(89.7 \%)$ & $<0.001$ \\
\hline Withdrawal of care & $41(16.5 \%)$ & $11(28.2 \%)$ & 0.079 \\
\hline
\end{tabular}

Bold indicate $p$ values $<0.05$.

${ }^{*}$ Categorical data are presented as number (\%) and continuous data as the mean.

$\mathrm{ICH}$, intracerebral haemorrhage; IVH, intraventricular haemorrhage.

$\mathrm{p}=0.079$ ). Within the EVD cohort, we found that there was greater proportion of patients within the mechanical compression group who had early withdrawal of care (under 7 days), versus those in the primary IVH subgroup (33.3\% vs $6.3 \% \mathrm{p}=0.024)$. However, the overall rate of withdrawal was the same between the two EVD groups $(33.3 \%$ vs $22.0 \% \mathrm{p}=0.497)$.

\section{Associations with poor outcome}

The mRS at discharge was significantly different between the EVD and non-EVD groups, with the portion of patients with a poor outcome (mRS 4-6) greater in the EVD group $(89.7 \%$ vs $52 \% \mathrm{p} \leq 0.001)$. Table 3 demonstrates univariate and multivariate comparisons of the whole cohort dichotomised to either good outcome or poor outcome based on mRS at discharge.

Analysis of the ICH location demonstrated that thalamic location was associated with poor outcome when compared with lobar or basal ganglia location. Higher ICH Scores were significantly associated with poor outcome when compared against lower ICH Scores. Presence of EVD, IVH and volume $\geq 30 \mathrm{~mm}^{3}$ was also significantly associated with poor outcome. In order to prevent over fitting of the model, only ICH location, ICH Score, presence of IVH and ICH volume were used in the multivariable logistic regression. Lobar location was associated with significantly decreased odds of poor outcome compared with thalamic location (OR $0.107-0.560)$. This model did not show presence of IVH and ICH volume was associated with poor outcome. Additional post-hoc exploratory multivariable analysis of whole group associations with poor outcomes was attempted using only thalamic location, ICH Score and presence of mechanical compression as input variables. This model demonstrated ICH Score as the only significant factor with poor outcome $(\mathrm{p} \leq 0.001)$; however, mechanical compression did trend towards significance $(\mathrm{p}=0.059)$.

Among the EVD subgroups, patients with mechanical compression had a significantly worse outcome than primary IVH patients $(100 \%$ vs $75 \% \mathrm{p}=0.037)$. Table 4 demonstrates univariate comparisons of the EVD cohort dichotomised to either good outcome or poor outcome. 
Table 2 Comparison of external ventricular drain (EVD) subgroups

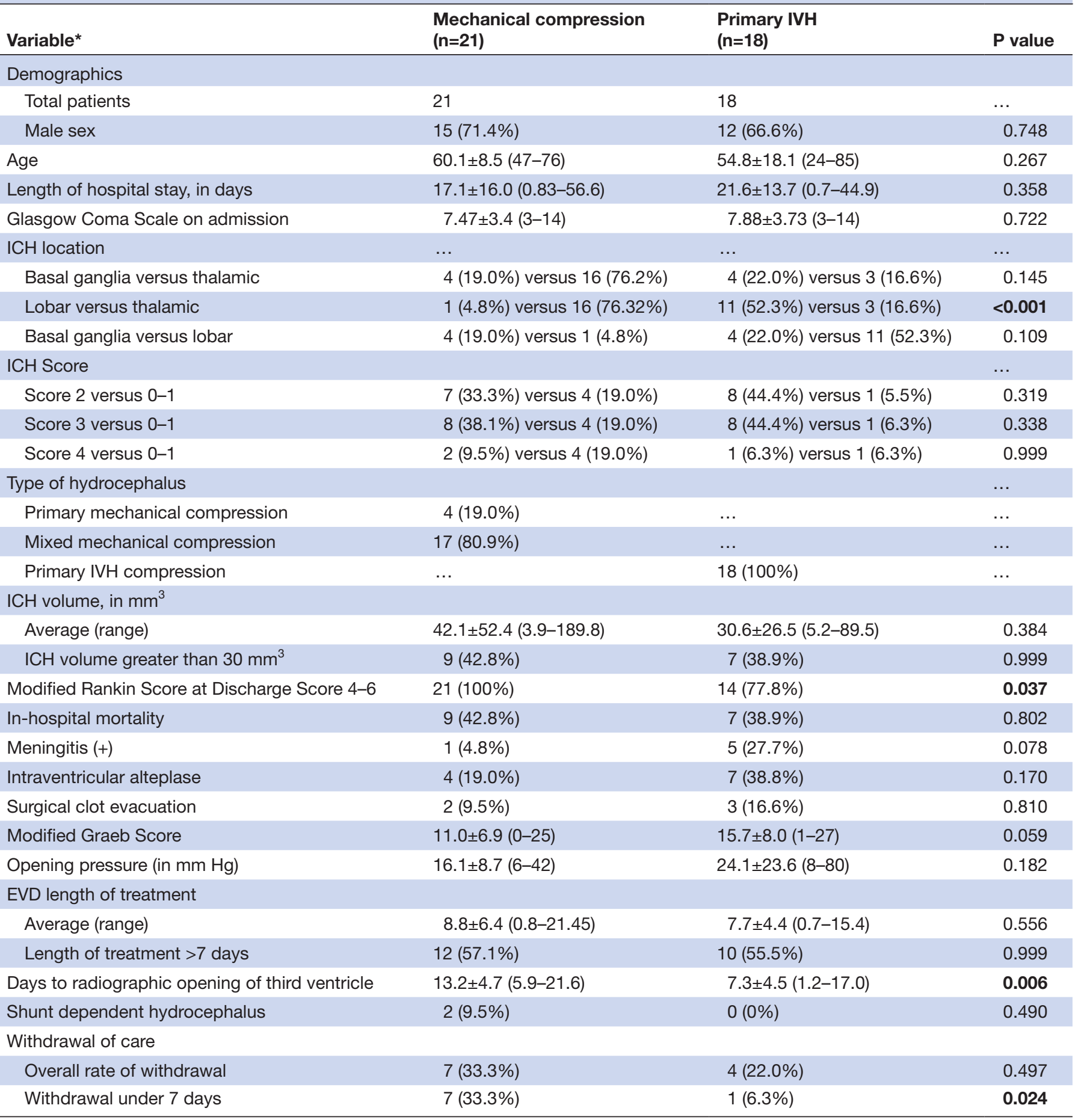

Bold indicate $\mathrm{p}$ values $<0.05$.

${ }^{*}$ Categorical data are presented as number (\%) and continuous data as the mean \pm standard deviation (range)

$\mathrm{ICH}$, intracerebral haemorrhage; IVH, intraventricular haemorrhage.

The other significant association with poor outcome within the EVD cohort includes thalamic location compared with lobar $(54.2 \%$ vs $25.7 \% \mathrm{p}=0.049)$, lower modified Graeb Score (12.6 vs $18.3 \mathrm{p}=0.01$ ), higher opening pressure $(20.6 \mathrm{~mm} \mathrm{Hg}$ vs $12.8 \mathrm{~mm} \mathrm{Hg} \mathrm{p=0.031)}$ and older age (60.0 vs $37.8 \mathrm{p}=0.006)$. ICH Score, ICH volume, meningitis and length of treatment with EVD were not associated with poor outcomes. Due to the smaller sample size of the EVD cohort, multivariable logistic regression models were not statistically appropriate.

Illustrative case

The patient presented with a $40 \mathrm{~mL}^{3}$ hypertensive thalamic haemorrhage with mixed compression hydrocephalus (figure 3A). Multiple attempts were made to clamp the EVD; however, the ventricles enlarged and 
Table 3 Whole group associations with poor outcome

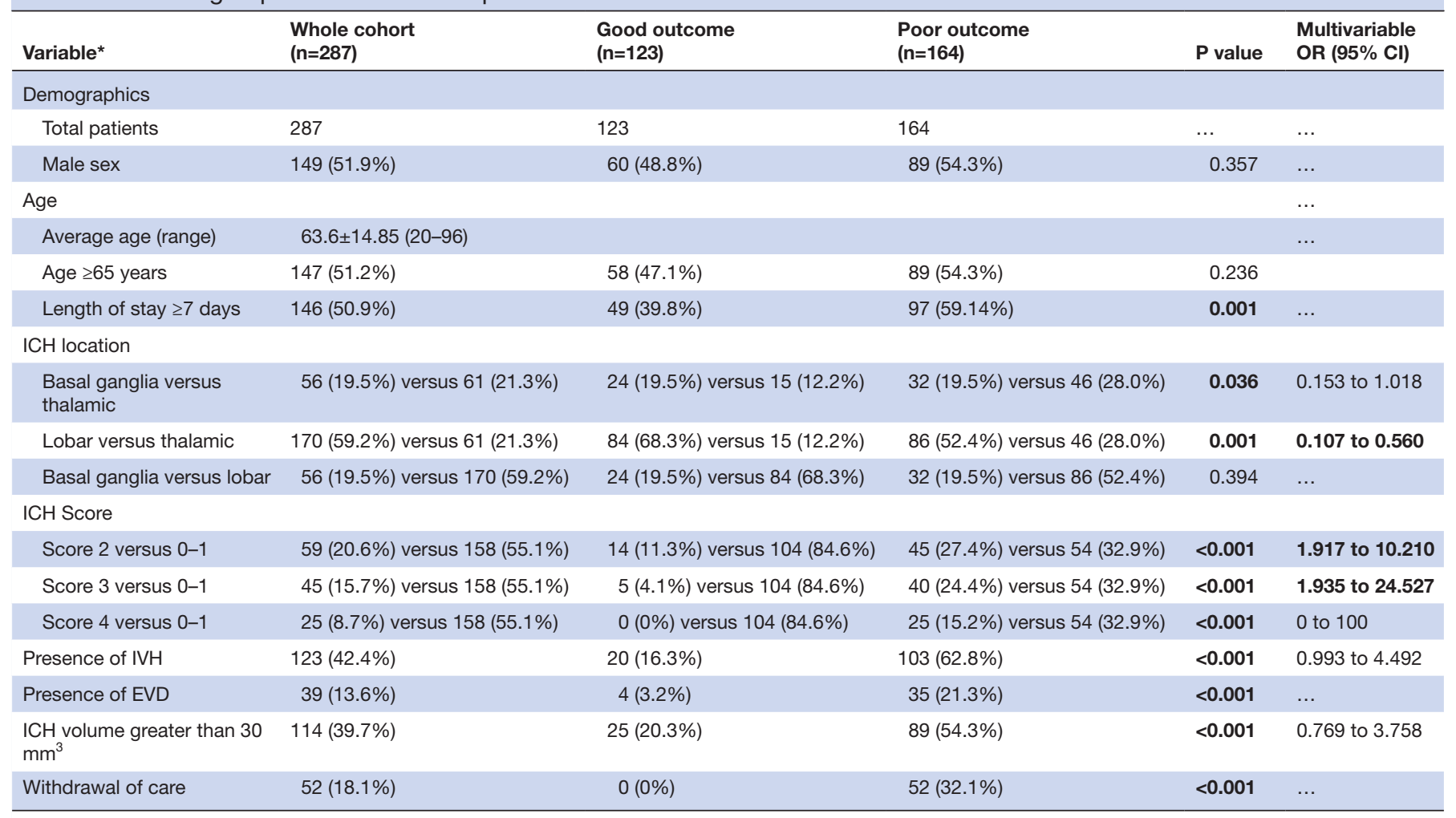

Bold indicate $p$ values $<0.05$.

${ }^{*}$ Categorical data are presented as number (\%).

EVD, external ventricular drain; ICH, intracerebral haemorrhage; IVH, intraventricular haemorrhage.

the exam deteriorated due to continued hydrocephalus. Targeted clot evacuation was therefore offered to attempt to decompress the ventricular outflow. Under general anaesthesia, a catheter was passed using stereotactic guidance. Clot aspiration was done with a $10 \mathrm{~mL}$ handheld syringe until first resistance. A postoperative CT was performed to confirm placement (figure 3B). Alteplase was administered directly into the clot through the catheter at $1.0 \mathrm{mg}$ in $1 \mathrm{~mL}$ followed by a $3 \mathrm{~mL}$ flush until the residual haematoma was less than $15 \mathrm{~mL}$. Figure 3C shows resolution of the haemorrhage after three doses of alteplase.

\section{DISCUSSION}

Patients who haemorrhage within the deep brain nuclei are known to have significantly worse outcomes compared with patients with similar sized peripherally located supratentorial ICH. Consistent with the results of this study, several other studies have shown that among patients who have an ICH that involves the thalamus has the highest rate of death or major disability. ${ }^{16-20}$ These poor outcomes have traditionally been explained primarily by the destruction of the vital thalamic nuclei and their associated central relay pathways. However, data from the secondary analysis of CLEAR III suggest that a unique set of patients with ICH and CSF flow obstruction have particularly poor outcomes in comparison to other cohorts with similar size and location. ICH within the deep nuclei has a unique anatomical position to cause obstruction to CSF flow by mechanical compression of the third ventricle, Foramen of Monroe, or cerebral aqueduct.

This is the first study to report the incidence of mechanical compression caused hydrocephalus after ICH. There was a total of $21(7.3 \%)$ patients that demonstrated a mixed or primary component of mechanical compression on initial CT imaging. Within the EVD group, the mechanical and mixed subgroups experienced significantly worse outcomes when compared with the primary IVH subgroup. These findings are consistent with the CLEAR III analysis demonstrating worse outcomes with mechanical obstruction, ${ }^{12}$ but has the advantage of evaluating a larger group of patients, not just those meeting the entry criteria for CLEAR III. Many of these patients included in this study would have been excluded from CLEAR based on the size of the haemorrhage. Although the modified Graeb Score was not significantly different between the EVD subgroups, the mechanical compression subgroup demonstrated a trend towards lower Scores. In the subgroup analysis of poor outcomes, a higher modified Graeb Score was associated with better outcomes, suggesting that within the EVD cohort-patients with greater volume of IVH may fare better than those with hydrocephalus and lower IVH volumes. The number of days to the radiographic opening of the third ventricle was demonstrated to be almost an entire week longer in 
Table 4 External ventricular drain (EVD) group associations with poor outcome

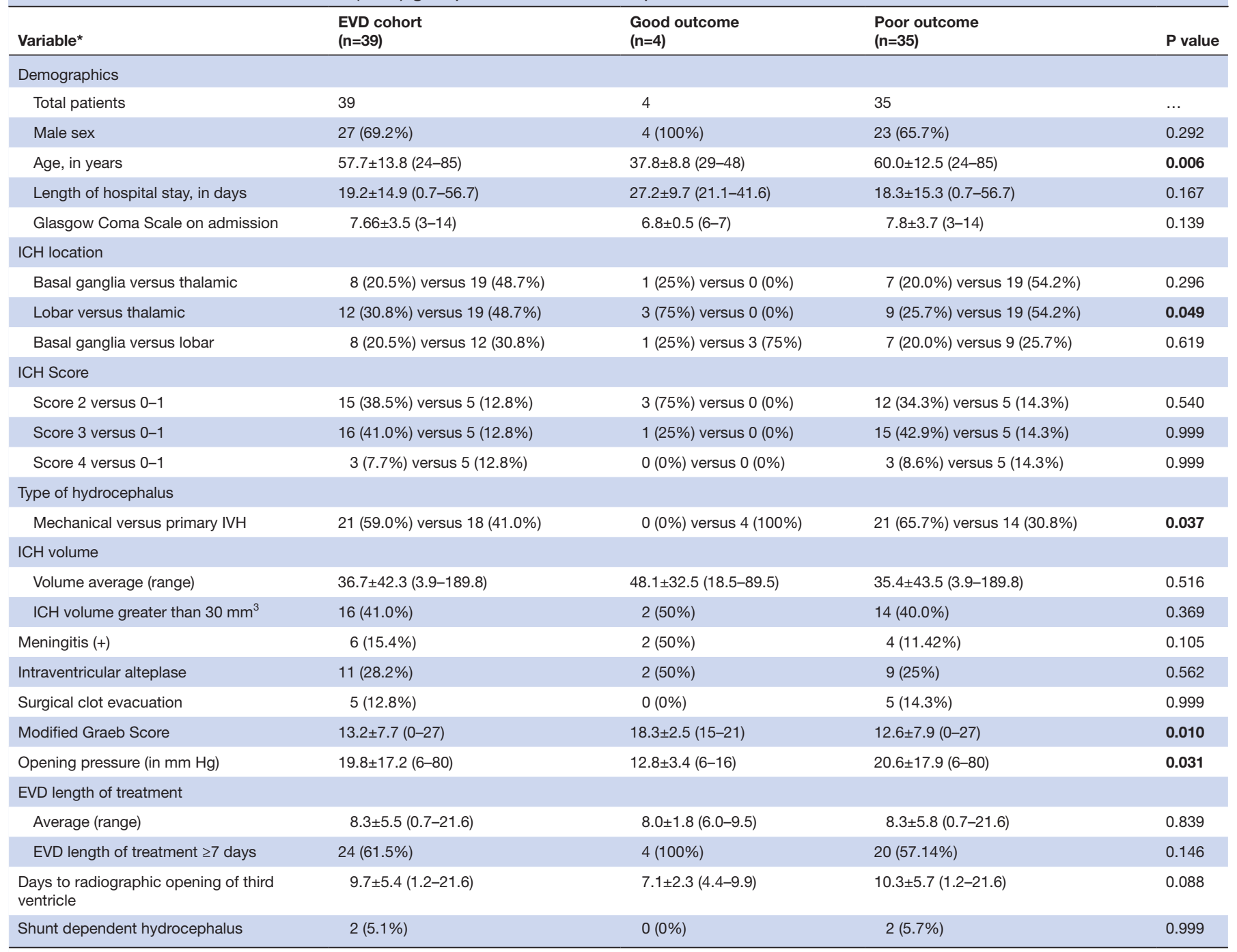

${ }^{*}$ Categorical data are presented as number (\%) and continuous data as the mean $\pm \mathrm{SD}$ (range). $\mathrm{ICH}$, intracerebral haemorrhage; IVH, intraventricular haemorrhage.
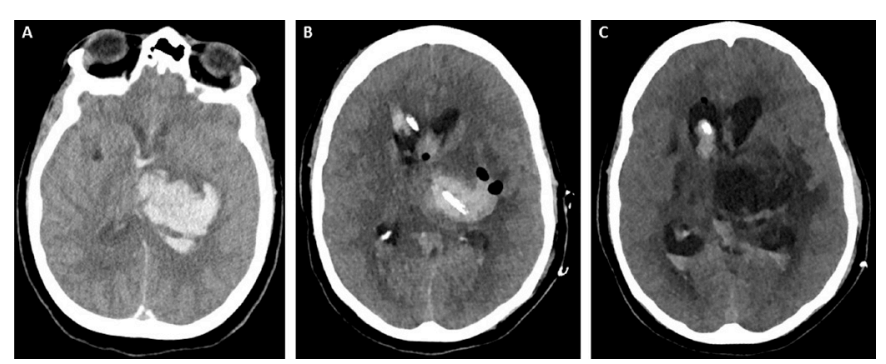

Figure 3 Illustrative case. (A) Initial non-contrasted CT head showing IPH in the deep grey structures with associated intraventricular haemorrhage resulting in mechanical obstruction of the third ventricle and aqueduct. (B) Interval placement of an external ventricular drain for treatment of hydrocephalus as well as placement of a catheter within the haemorrhage. (C) Resolution of the intraparenchymal haemorrhage after treatment with alteplase. IPH, Intraparenchymal hemorrhage. the mechanical compression subgroup when compared with the IVH subgroup, further supporting the hypothesis that mechanical compression hydrocephalus may be caused by clot burden obstructing ventricular patency.

Consistent with the literature, the EVD group had significantly worse outcomes than the non-EVD group. Multivariable logistic regression model of this group demonstrated that $\mathrm{ICH}$ volume $\geq 30 \mathrm{~mm}^{3}$ and presence of IVH do not adequately explain the outcome differences, but location may play an important role in prediction of poor outcomes. Additional multivariable analysis did not demonstrate mechanical compression as an independent predictor of poor outcome within the whole cohort of patients with ICH; however, this may have been due to the presence of type II error and rarity of mechanical compression within the larger group. Mechanical compression was seen as univariate predictor of poor outcome within the EVD subgroup analysis which is 
consistent with the findings of the alteplase treated group shown by Ullman et al CLEAR III post-hoc analysis. Comparisons of the EVD subgroups demonstrated a significantly higher portion of thalamic haemorrhages in the mechanical compression subgroup and a higher rate of meningitis in the IVH subgroup. However, the only significant associations with poor outcome in the EVD subgroups were age, lower modified Graeb Scores, opening pressure, thalamic location and mechanical compression type hydrocephalus. It is unknown if the poor outcomes seen in the EVD subgroups were primarily due to predominance of deep haemorrhages, mechanical compression of ventricular outflow or some combination of the two. Further studies should be done to examine if a causal relationship between mechanical compression and stroke outcomes can be differentiated from the effects of haemorrhage within the deep nuclei.

\section{Limitations}

Withdrawal of care was noted $52(88.1 \%)$ of patients who died in hospital, with $41(65.5 \%)$ undergoing a change of code status while hospitalised. Due to the retrospective nature of this study and the high levels of withdrawal of care, caution should be used in interpreting these data as prognostic. Due to small sample size in EVD cohort (table 4), the tendency for type II error may be high, and the lack of significance of some variables may be due to decreased power to detect a difference between groups. The long-term modified Rankin Disability Scores beyond discharge were unable to be assessed due to the retrospective nature of this study. Lastly, this study was conducted from patients primarily funded by Medicare and Medicaid in the (REGIONAL LOCATION OF INSTITUTION BLINDED) and may not be generalisable to all populations.

\section{Conclusions}

This is the first study to report the incidence of mechanical compression hydrocephalus after ICH. Of all patients with spontaneous supratentorial ICH, 7.3\% demonstrated a mixed or primary component of mechanical compression EVD dependent hydrocephalus on CT imaging. This group also experienced significantly worse outcomes and delayed opening of the third ventricle when compared with the primary IVH hydrocephalus subgroup. It is unknown if the worse morbidity in mechanical compression subjects is purely related to damage to deep structures surrounding the third ventricle or if secondary damage from hydrocephalus could be mitigated with targeted minimally invasive clot evacuation.

Contributors B0: design of the study, data acquisition, analysis, interpretation of results, and drafting of manuscript. OA and MT: interpretation of results, and drafting of manuscript. HD: analysis, interpretation of results, drafting of manuscript. APC: design of the study, analysis, interpretation of results and drafting of manuscript.

Funding The authors have not declared a specific grant for this research from any funding agency in the public, commercial or not-for-profit sectors.

Competing interests None declared.
Patient consent for publication Not required.

Ethics approval This study complies with the guidelines for human studies and was conducted ethically in accordance with the World Medical Association Declaration of Helsinki. Approval by the site-specific Institutional Review Board was obtained (UNM HRPO 18-374). The data collection involved no risk to participants and patient identifiers were not used, a waiver for consent was granted.

Provenance and peer review Not commissioned; externally peer reviewed. Data availability statement Data are available upon reasonable request. Deidentified study data can be made available upon reasonable request to the corresponding author.

Open access This is an open access article distributed in accordance with the Creative Commons Attribution Non Commercial (CC BY-NC 4.0) license, which permits others to distribute, remix, adapt, build upon this work non-commercially, and license their derivative works on different terms, provided the original work is properly cited, appropriate credit is given, any changes made indicated, and the use is non-commercial. See: http://creativecommons.org/licenses/by-nc/4.0/.

ORCID iD

Bryce Owen http://orcid.org/0000-0002-0058-9500

\section{REFERENCES}

1 Benjamin EJ, Virani SS, Callaway CW, et al. Heart disease and stroke Statistics -2018 update: a report from the American heart association. Circulation 2018;137:426.

2 Andersen KK, Olsen TS, Dehlendorff C, et al. Hemorrhagic and ischemic strokes compared: stroke severity, mortality, and risk factors. Stroke 2009;40:2068-72.

3 González-Pérez A, Gaist D, Wallander M-A, et al. Mortality after hemorrhagic stroke: data from general practice (the health improvement network). Neurology 2013;81:559-65.

4 Hemphill JC, Greenberg SM, Anderson CS, et al. Guidelines for the management of spontaneous intracerebral hemorrhage: a guideline for healthcare professionals from the American heart Association/ American stroke association. Stroke 2015;46:2032-60.

5 McKissock W, Richardson A, Taylor J. Primary intracerebral haemorrhage: a controlled TRAIL of surgical and conservative treatment in 180 unselected cases. The Lancet 1961;278:221-6.

6 Awad IA, Polster SP, Carrión-Penagos J, et al. Surgical performance determines functional outcome benefit in the minimally invasive surgery plus recombinant tissue plasminogen activator for intracerebral hemorrhage evacuation (MISTIE) procedure. Neurosurgery 2019;84:1157-68.

7 Gregson BA, Rowan EN, Mitchell PM, et al. Surgical trial in traumatic intracerebral hemorrhage (STITCH(Trauma)): study protocol for a randomized controlled trial. Trials 2012;13.

8 Mendelow AD, Gregson BA, Rowan EN, et al. Early surgery versus initial conservative treatment in patients with spontaneous supratentorial lobar intracerebral haematomas (STICH II): a randomised trial. The Lancet 2013;382:397-408.

9 Mould WA, Carhuapoma JR, Muschelli J, et al. Minimally invasive surgery plus recombinant tissue-type plasminogen activator for intracerebral hemorrhage evacuation decreases perihematomal edema. Stroke 2013;44:627-34.

10 ENRICH: Early MiNimally-invasive Removal of IntraCerebral Hemorrhage (ICH) - Full Text View - ClinicalTrials.gov. Available: https://clinicaltrials.gov/ct2/show/NCT02880878 [Accessed March 30, 2020].

11 Zheng J, Li H, Guo R, et al. Minimally invasive surgery treatment for the patients with spontaneous supratentorial intracerebral hemorrhage (MISTICH): protocol of a multi-center randomized controlled trial. BMC Neurol 2014;14.

12 Hanley DF, Lane K, McBee N, et al. Thrombolytic removal of intraventricular haemorrhage in treatment of severe stroke: results of the randomised, multicentre, multiregion, placebo-controlled CLEAR III trial. The Lancet 2017;389:603-11.

13 Ullman NL, Tahsili-Fahadan P, Thompson CB, et al. Third ventricle obstruction by thalamic intracerebral hemorrhage predicts poor functional outcome among patients treated with alteplase in the clear III trial. Neurocrit Care 2019;30:380-6.

14 Hemphill JC, Bonovich DC, Besmertis L, et al. The ICH score: a simple, reliable grading scale for intracerebral hemorrhage. Stroke 2001;32:891-7.

15 Morgan TC, Dawson J, Spengler D, et al. The modified Graeb score: an enhanced tool for intraventricular hemorrhage measurement and prediction of functional outcome. Stroke 2013;44:635-41. 
16 Delcourt C, Sato S, Zhang S, et al. Intracerebral hemorrhage location and outcome among INTERACT2 participants. Neurology 2017;88:1408-14.

17 Neisewander BL, Hu K, Tan Z, et al. Location of thalamic hemorrhage impacts prognosis. World Neurosurg 2018;116:e525-33.

18 Zacharia BE, Vaughan KA, Hickman ZL, et al. Predictors of longterm shunt-dependent hydrocephalus in patients with intracerebral hemorrhage requiring emergency cerebrospinal fluid diversion. Neurosurg Focus 2012;32:E5.
19 Chen C-C, Liu C-L, Tung Y-N, et al. Endoscopic surgery for intraventricular hemorrhage (IVH) caused by thalamic hemorrhage: comparisons of endoscopic surgery and external ventricular drainage (EVD) surgery. World Neurosurg 2011;75:264-8.

20 Eslami V, Tahsili-Fahadan P, Rivera-Lara L, et al. Influence of intracerebral hemorrhage location on outcomes in patients with severe intraventricular hemorrhage. Stroke 2019;50:1688-95. 